\title{
Appropriateness of Tibial Rotation for Isolation of the Medial and Lateral Hamstrings
}

\author{
1,2 Wootaek Lim* \\ ${ }^{1}$ Department of Physical Therapy, Rehabilitation Science Faculty, Woosong University, Daejeon, \\ Republic of Korea. ${ }^{2}$ Woosong Institute of Rehabilitation Science, Rehabilitation Science Faculty, \\ Woosong University, Daejeon, Republic of Korea.
}

Submitted 13 July 2020; Accepted in final form 13 September 2020.

\begin{abstract}
Background. It is unclear whether the clinical use of tibial rotation to measure the strength of the medial hamstrings and lateral hamstrings in isolation is based on sound scientific evidence or is simply based on anatomical features. Objectives. To verify the appropriateness of tibial rotation for isolation of the medial hamstrings and lateral hamstrings, the participants performed neutral rotation (NR), external rotation (ER), and internal rotation (IR). Methods. Forty-six young and healthy adults (age $21.9 \pm 2.2$ years, height $167.9 \pm 8.6 \mathrm{~cm}$, the weight of $63.8 \pm 13.9$ $\mathrm{kg}$ ) participated in the study. The electromyography (EMG) activity and knee flexion torque were measured and compared among different postures; NR, ER, and IR. Results. The Max of the EMG activity of the biceps femoris long head, semitendinosus, and semimembranosus muscles was significantly reduced in ER and IR compared with that in NR. There were no significant differences in muscle activity between ER and IR. Knee flexion force and torque were significantly reduced in IR compared with those in NR and ER. EMG activity was significantly diminished during tibial rotation compared with that in NR. Conclusion. Contrary to the general assumption, IR and ER had a limited influence on the isolation of medial hamstrings and lateral hamstrings; thus, they may be unnecessary for medial hamstrings and lateral hamstrings normalization. The uniform training of the hamstring muscles in NR for MVIC may be more appropriate than isolating the muscles in ER and IR.
\end{abstract}

KEYWORDS: Electromyography, Hamstring Muscles, Muscle Strength, Rotation.

\section{INTRODUCTION}

The hamstring is divided into the $\mathrm{MH}$ and $\mathrm{LH}$ according to the anatomical course and attachment of the muscle. The MH consists of the semitendinosus (ST) and semimembranosus (SM) muscles, and the LH consists of the long (LHBF) and short head of the biceps femoris (SHBF). Owing to their anatomical positions, the $\mathrm{MH}$ is known to assist the internal rotation (IR). In contrast, the LH assists the external rotation (ER) of the tibia in an open-kinetic chain. Because the $\mathrm{MH}$ and $\mathrm{LH}$ are antagonistic in tibial rotation, textbooks recommend independent measurement and assessment of the $\mathrm{MH}$ and $\mathrm{LH}$ for manual muscle testing (MMT) $(1,2)$. This is also why, in clinical practice, specialized exercise programs are designed for each of the hamstring muscles instead of including all of them into one exercise program. However, it is unclear whether the application of tibial rotation is rooted in scientific evidence or is based on mere assumptions (i.e., bias) based on anatomical features.

There have been various attempts to identify the differences in the EMG activities of the lower-

*. Corresponding Author:

Wootaek Lim, PhD

E-mail: wootaeklimpt@wsu.ac.kr 
limb muscles about postural change (3). A study comparing the vastus medialis and vastus lateralis, which comprise the quadriceps, vastus medialis activity was significantly elevated during adduction compared with during IR, ER, and abduction (4-6). Concerning the hamstring, which has a posterior position on the thigh, multiple studies have examined the effects of changes of hip and knee joint angles on the EMG activity of the $\mathrm{MH}$ and $\mathrm{LH}$ and the changes in resistance (7-9). However, there were limitations in studies investigating how much tibial rotation influences the EMG activity of the MH and LH. This is associated with the knee flexion force, a commonly used parameter in clinical practice.

In clinical practice, the strength of the $\mathrm{MH}$ and LH is measured and assessed in isolation before and after treatment based on the MMT posture suggested in textbooks $(1,2)$. However, universal use cannot serve as scientific evidence. Sound evidence from experiments is needed. As generally inferred, if muscle activity is elevated or diminished according to the tibial rotation, normalizing other values based on the maximum value can be used as critical evidence in developing effective treatment plans. The present study measured and comparatively analyzed the EMG activity and knee flexion force in three postures neutral rotation (NR), ER, and IR-for the isolation of the LHBF, ST, and SM, which comprise the $\mathrm{MH}$ and $\mathrm{LH}$.

\section{MATERIALS AND METHODS}

Participants. Forty-six healthy young adults were enrolled. The participants had a mean age of $21.9 \pm 2.2$ years, the height of $167.9 \pm 8.6 \mathrm{~cm}$, and weight of $63.8 \pm 13.9 \mathrm{~kg}$ (Table 1). The included participants did not have any disease, injury, deformity, or history of surgery of the lower back and knee joint. Those who had pain in the relevant areas in the last six months were excluded from the study. Informed consent was obtained from all participants. This study was approved and monitored by the Institutional Review Board.



Figure 1. Maximal Voluntary Isometric Contraction of the Hamstrings in Prone Position.

Protocol. The participants laid on the treatment table in the prone position with their knee joint bent $60^{\circ}(8,10-12)$. The pelvis and contralateral lower extremity were fixed to the table with a belt. Three trials of maximal voluntary isometric contraction (MVIC) were performed for each posture $(5 \mathrm{~s} /$ trial, 30 -s rest between trials). To prevent fatigue, the participants rested for 2 min after performing three trials of MVIC in one posture before proceeding to the next posture $(13,14)$. Resistance to movement during MVIC was provided using a strap, and EMG and knee flexion force values were recorded in real-time using the Delsys EMG system (Delsys Inc., Boston, MA, USA) and the wireless strain gauge (Re-live Inc., Kimhae, Korea) placed on the middle of the strap, respectively (15). To prevent movement during contraction, one end of the strap was tightly fixed $3 \mathrm{~cm}$ below the 
lateral malleolus and the other end to a fixed pillar. To calculate the torque, the strap was vertically placed against the lower leg (Figure 1 ). The torque was calculated by multiplying the force $(\mathrm{N})$ by the distance $(\mathrm{m})$ from the lateral epicondyle of the femur to the ankle strap (16). Three postures were used (NR, IR, ER) in random order. For the LHBF, the examiner provided downward and inward resistance when the participant flexed the knees after the tibia was externally rotated by $30^{\circ}$. The toes were facing outward with the knees bent $60^{\circ}$. The examiner instructed the participant to keep the tip of the toe facing outward to maintain the ER during knee flexion. For measuring MVIC for the ST and $\mathrm{SM}$, the tibia was internally rotated by $30^{\circ}$ during knee flexion, and resistance was provided in downward and outward directions. From the root mean square (RMS) measured at three different postures, the highest RMS was set to 100 , and the other two were computed and presented as \%Max.

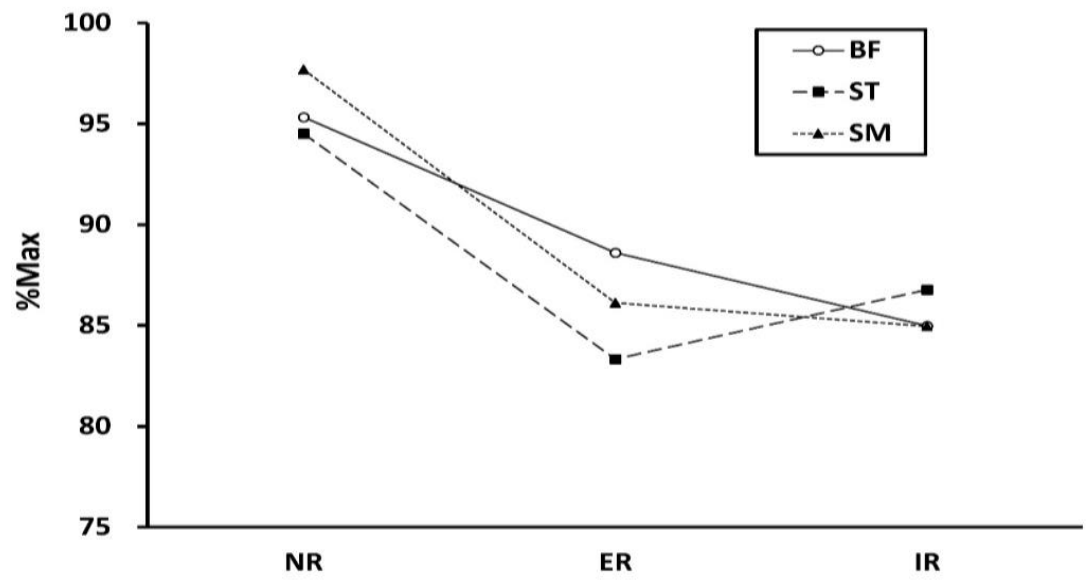

Figure 2. Changes of Electromyographic Activities of the Hamstring Muscles According to Tibial Rotation. BF = biceps femoris, $\mathrm{ST}=$ semitendinosus, $\mathrm{SM}=$ semimembranosus, $\mathrm{NR}=$ neutral rotation, ER, external rotation, $\mathrm{IR}=$ internal rotation.

Table 1. Characteristics of the Participants

\begin{tabular}{lccc}
\hline Gender & Age $(\mathbf{y})$ & Weight $(\mathbf{k g})$ & Height $(\mathbf{c m})$ \\
\hline Male $(\mathbf{n}=\mathbf{2 4})$ & $22.4 \pm 2.8$ & $72.8 \pm 12.2$ & $174.3 \pm 5.0$ \\
Female $(\mathbf{n}=\mathbf{2 2})$ & $21.3 \pm 1.0$ & $54.1 \pm 7.7$ & $160.9 \pm 5.8$ \\
\hline
\end{tabular}

Data ia table are presented as mean \pm standard deviation.

Table 2. Reliability of Electromyographic Activity and Knee Flexion Force Measurements Between the $1^{\text {st }}$ and $2^{\text {nd }}$ Trials

\begin{tabular}{lcccc}
\hline & Biceps femoris & Semitendinosus & Semimembranosus & Force \\
\hline Neutral rotation & 0.960 & 0.916 & 0.956 & 0.885 \\
External rotation & 0.907 & 0.911 & 0.914 & 0.832 \\
Internal rotation & 0.949 & 0.947 & 0.889 & 0.871 \\
\hline
\end{tabular}

Table 3. Changes of Knee Flexion Force and Torque According to Tibial Rotation

\begin{tabular}{lcc}
\hline & Force $(\mathbf{N})$ & Torque $(\mathbf{N m})$ \\
\hline Neutral rotation & $133.8 \pm 59.3$ & $53.1 \pm 27.3$ \\
External rotation & $136.4 \pm 54.9$ & $53.9 \pm 24.7$ \\
Internal rotation & $117.9 \pm 47.6$ & $46.6 \pm 21.9$ \\
\hline
\end{tabular}

Data are presented as mean \pm standard deviation.

Surface EMG signals were collected from the LHBF, ST, and SM using a Trigono ${ }^{\mathrm{TM}}$ wireless EMG system. Delsys EMGworks ${ }^{\circledR}$ Acquisition and Analysis software were used to analyze the
EMG signals. Raw EMG data were sampled at $2000 \mathrm{~Hz}$. Then, the signals were processed into the RMS with a window of $125 \mathrm{~ms}$. A $20-450-\mathrm{Hz}$ band-pass filter was used in this study, together 
with a $60-\mathrm{Hz}$ notch filter. The EMG electrodes were placed on selected hamstrings by the SIAM (Surface Electromyography for Non-invasive Assessment of Muscles) guidelines and the Anatomical Guide for the Electromyography textbook $(17,18)$. Knee flexion force was measured simultaneously with electromyography using a wireless strain gauge at a frequency of $4 / \mathrm{s}$ (once every $0.25 \mathrm{~s}$ ). For the storage of data, the measurements were transmitted to a laptop.

Statistical Analysis. The reliability of the three measurements of the RMS of the EMG activity and knee flexion force of the hamstring was analyzed using intraclass correlation coefficients (ICC 2,1). The RMS of the EMG activity for each muscle was converted to \% Max. The differences among three postures (NR, ER, IR) were analyzed using one-way repeatedmeasures analysis of variance (ANOVA). Additionally, the Bonferroni test was performed as a post-hoc test for pairwise comparisons. The knee flexion force and torque values among the three postures were also compared using one-way repeated-measures ANOVA. Statistical analysis was done with IBM SPSS Statistics 23 for windows (IBM Corp., Armonk, NY, USA). Statistical significance was set at $\mathrm{p}<0.05$. The sample size was calculated using the G*Power version 3.1.9.7 software (Heinrich- HeineUniversität Düsseldorf, Düsseldorf, Germany).

\section{RESULTS}

Both the reliability of the RMS values of the LHBF, SM, and ST during three trials of MVIC in one posture and the reliability of the knee flexion force of the hamstrings during three trials of MVIC in one posture were high. The ICC for RMS was 0.9 or less than but close to 0.9 for the following comparisons: $1^{\text {st }}$ vs. $2^{\text {nd }}$ trial (Table 2), $2^{\text {nd }}$ vs. $3^{\text {rd }}$ trial, and $3^{\text {rd }}$ vs. $1^{\text {st }}$ trial $(\mathrm{P}<0.001)$.

Concerning the \%Max, which indicates whether tibial rotation influences the change of the activity of the involved muscle, the percentage significantly decreased for the $\operatorname{LHBF}(\mathrm{P}=0.004)$, ST $(\mathrm{P}<0.001)$, and SM $(\mathrm{P}<0.001)$ during ER compared with NR (Figure 2). Compared with that during $\mathrm{NR}$, the \% Max also significantly decreased for the LHBF $(\mathrm{P}<0.001)$, ST $(\mathrm{P}=$ 0.015), and SM $(\mathrm{P}<0.001)$ during IR. Between $\mathrm{ER}$ and IR, there were no significant differences in the LHBF $(\mathrm{P}=0.548), \mathrm{ST}(\mathrm{P}=0.372)$, and SM $(\mathrm{P}=0.903)$.

Both knee flexion force and torque were significantly reduced during IR: NR vs. IR ( $<$
0.001) and ER vs. IR $(\mathrm{P}<0.001)$ (Table 3). There were no significant differences between NR and $\mathrm{ER}(\mathrm{P}=1.000)$.

\section{DISCUSSION}

In clinical practice, individual muscles need to be measured and assessed in isolation to identify a patient's problem accurately, which enables selective training. Thus, there are recommended postures for individual muscle assessment during MMT. For the lower limbs, tibial rotation is required in the prone knee bending position for the selective training of the $\mathrm{MH}$ and $\mathrm{LH}$. However, whether the utility of tibial rotation is scientifically proven or is simply a bias based on the anatomical features of the hamstring has not been established. In the present study, quantitative measurements were taken using EMG and a tension gauge for scientific verification. The reliability of the experiment was established, with high ICC values obtained for all measurements.

Theoretically, the LHBF, which is a part of the LH, shows elevated activity in ER as an external rotator of the knee joint and relatively diminished activity in IR. Further, the ST and SM, both of which are a part of the MH, should show antagonistic actions. However, in the actual experiment, a contradictory pattern was observed when examining the differences of EMG activity among the LHBF, ST, and SM, according to the tibial rotation. One notable finding is that all three muscles showed statistically similar patterns. EMG activity was significantly reduced in ER and IR compared with that in NR. More specifically, the LHBF, which functions as an external rotator, showed a $7.4 \%$ activity reduction in ER than in NR.

Meanwhile, the ST and SM, which function as internal rotators, showed an activity reduction of $11.0 \%$ and $11.5 \%$, respectively, in ER compared with that in NR. Although the ST and SM showed higher activity reductions than the LHBF in ER, the difference was not substantial, contrary to clinical expectations. The \% Max was the highest in NR for all muscles, and the percentage decreased during tibial rotation regardless of direction. This suggests that results contrary to the expected outcome may be obtained when tibial rotation is performed for the purpose of selective training in clinical practice. In some studies on tibial rotation, the MH showed elevated EMG activity during IR, and the LH showed increased EMG activity during ER, consistent with the 
general assumption (19). However, the resistance used was very low (only $5 \%$ of body weight), and the same results may not be obtained in the clinical setting when high-intensity resistance training is performed. In two different studies, similar to this study, the maximal isometric contraction was performed. In a study by Jónasson, the Influence of tibial rotation on EMG activity of hamstrings were analyzed, though the activity was not measured in NR (20). MH versus LH activation ratio was significantly lesser in ER than IR, but there was no difference in the ratio in men and women when analyzed separately. A recent study by Beyer also showed a decrease in MH activity in IR and ER, and no significant difference in $\mathrm{LH}$ at $60^{\circ}$ knee flexion (21). In the present study, in which maximal isometric contraction was applied, the EMG activities of all three muscles were significantly diminished in ER and IR compared with those in NR. However, the differences in the maximum and minimum values for each posture were not large, with $10.7 \%$ for LHBF, $11.0 \%$ for ST, and $13.3 \%$ for SM. On the basis of findings in this study and those of previous studies, even if hamstring isolation is somewhat possible with tibial rotation, it may have low practical value for patients because low-intensity muscle training has little benefits. Further, the isolation of particular muscles through tibial rotation may be limited, as found in this study.

Many clinicians have readily accepted and widely used the posture for MMT recommended by Daniels and Worthingham or Kendall and McCreary in their textbooks $(1,2)$. However, these two textbooks show disparities for some muscles, leading to different results (22). In other words, the current posture widely used for MMT should be scientifically tested instead of being blindly accepted. Like this study, some other studies have reported that it is difficult to isolate a specific muscle from its synergist during maximal isometric contraction. Perry performed the gastrocnemius standing heel-rise test to isolate the gastrocnemius from calf muscles consisting of superficial (gastrocnemius) and deep muscles (soleus). However, as the EMG activity of the soleus increased to $87 \%$ of the maximum, the gastrocnemius could not be isolated during MMT (23). In another experiment, nine MMTs were performed, including the scapular protraction muscle test, to isolate the serratus anterior, which is essential for scapular stability. However, maximum serratus anterior activity was observed in several MMTs, not in a single MMT.

Furthermore, in the scapular protraction test, which is the most commonly used test, the EMG activity was lower than in other MMTs (24). The rhomboid is another muscle essential for scapular stability, and multiple attempts have been made to isolate this muscle from other muscles owing to its significance. In the three classic rhomboids MMTs, the rhomboid could not be isolated from its synergists such as the posterior deltoid, levator scapulae, and middle trapezius during MVIC (25). Another study attempted to differentiate various muscles mobilized for shoulder stability (e.g., supraspinatus, infraspinatus, trapezius, deltoid) using 15 MMTs. However, it was difficult to isolate the muscles, as several muscles were maximally activated simultaneously in most MMT postures (26). For the lower limbs, one study examined changes in the EMG activity of the $\mathrm{MH}$ and $\mathrm{LH}$ according to the tibial rotation, similar to this study. The EMG activities of the $\mathrm{MH}$ and $\mathrm{LH}$ were measured during maximal isometric contraction with the knee joint bent $70^{\circ}$. Although the degree of statistical differences varied across groups, the degree of reduction caused by tibial rotation was small (14\% for the ST, $18 \%$ for the SM, and $17 \%$ for the BFLH) (27). However, the findings have limited clinical value and must be interpreted with caution because muscle force was not measured during the experiment. In the present study, knee flexion force was also quantified in addition to EMG activity for each muscle.

Although EMG is an excellent tool for muscle activation analysis, it does not show the force or torque created during muscle activation $(28,29)$. In the current study, it was able to measure the knee flexion force in real-time during maximal isometric contraction by attaching a tension gauge in the middle of the strap over the ankle. Torque, which was calculated by multiplying by individual moment arm, was partially inconsistent with other EMG values. The \%Max was significantly reduced in ER and IR compared with that in NR; however, it did not significantly differ between ER and IR. Meanwhile, force and torque were significantly reduced in IR compared with those in both NR and ER, with no significant difference between NR and ER. This was consistent with a previous study, which showed a significant decrease in peak force output (21). Although EMG activity and force seem inconsistent with each 
other in this study, EMG activity was also partially diminished in IR. There were no significant differences in \%Max between ER and IR; however, the $\mathrm{BF}$ and $\mathrm{SM}$ showed minimum values (85.3\% and $84.6 \%$, respectively) in IR. The ST reached the minimum value in ER; however, the frequency of minimum values by posture, instead of the mean, for each muscle was $26.6 \%$ in IR and $15.6 \%$ in ER, showing a higher frequency of minimum values in IR.

A single factor and multiple causes cannot explain the significant reduction of force in IR. First, the reduction of force in IR results from the involvement of all muscles related to knee flexion instead of only the three muscles measured in this experiment. Muscles involved in knee flexion other than the three muscles in this study cocontracted as synergists and led to the final knee flexion force (30). Second, some muscles change in length during tibial rotation, and a change of muscle length means that the muscle force could be reduced owing to the length-tension relationship $(8,31)$. Considering that the lowest force was observed in IR, it is possible that the sarcomere length for efficient muscle contraction is not achieved in ER and IR compared with NR. Finally, muscles can lose or gain force depending on the change in their course. There is no loss of force in the direction of a muscle, and the bone where it is inserted makes a $90^{\circ}$ angle. However, if this angle is changed as a result of a change of insertion during longitudinal axis rotation, the absolute value of the force of the individual muscle is not altered, but some of the force is diverted horizontally; therefore, the vertical force (on the actual bone movement) may be diminished. The site of muscle insertion is altered during tibial rotation, which changes the direction of the muscle course. Some muscles involved in this action will gain force, while some will lose force. Furthermore, because tibial rotation alters muscle insertion, it will also alter the length of the MA, the distance between a joint axis and the line of force acting on the joint, and ultimately will affect the torque that rotates the joint.

Several limitations to this study need to be acknowledged. Since only healthy young adults participated, it is difficult to generalize. Responses might vary with age and in individuals experiencing pain. Future investigations are necessary to validate these. Additionally, the current study has only examined the EMG activity and forces. Mobility of knee joint allowing tibial rotation depends on the individual, and this property might affect EMG activities and muscle forces.

\section{CONCLUSION}

Tibial rotation is widely used in clinical practice for isolating individual hamstring muscles for their measurement, assessment, and normalization. However, findings in this study showed that the EMG activity of both the MH and LH is diminished in IR and ER compared with that in NR. Additionally, the knee flexion force was also reduced in IR. Besides providing limited isolation of the $\mathrm{MH}$ and $\mathrm{LH}$, tibial rotation may also not have substantial benefits for the selective training of these muscles.

\section{APPLICABLE REMARKS}

- It is suggested that uniform assessment and rehabilitation for the $\mathrm{MH}$ and $\mathrm{LH}$ in NR may be efficient in the clinical (or sports) setting, requiring maximum or equivalent highintensity resistance training.

\section{FUNDING}

A Basic Science Research Program supported this study through the National Research Foundation of Korea (NRF) funded by the Ministry of Education (NRF2017R1C1B5076885) and 2020 Woosong University Academic Research Funding.

\section{CONFLICT OF INTEREST}

The author has no conflict of interest to disclose.

\section{REFERENCES}

1. Kendall FP, McCreary EK, Provance PG, Rodgers MM, Romani WA. Muscles: Testing and Function, with Posture and Pain. 5th ed.: Baltimore, MD: LWW; 2005.

2. Avers D, Brown M. Daniels and Worthingham's Muscle Testing: Techniques of Manual Examination and Performance Testing. 10th ed.: Saunders; 2018.

3. Fiebert IM, Spielholz NI, Applegate EB, Carbone M, Gonzalez G, Gorack WM. Integrated EMG study of the medial and lateral heads of the gastrocnemius during isometric plantar flexion with varying cuff weight loads. $J$ Back Musculoskelet Rehabil. 1998;11(1):19-26. doi: 10.3233/BMR-1998-11103 pmid: 24572383

4. Hanten WP, Schulthies SS. Exercise effect on electromyographic activity of the vastus medialis oblique and vastus lateralis muscles. Phys Ther. 1990;70(9):561-565. doi: 10.1093/ptj/70.9.561 pmid: 2392485 
5. LeVeau BF, Rogers C. Selective training of the vastus medialis muscle using EMG biofeedback. Phys Ther. 1980;60(11):1410-1415. doi: 10.1093/ptj/60.11.1410 pmid: 7433520

6. Mirzabeigi E, Jordan C, Gronley JK, Rockowitz NL, Perry J. Isolation of the vastus medialis oblique muscle during exercise. Am J Sports Med. 1999;27(1):50-53. doi: 10.1177/03635465990270011601 pmid: 9934418

7. Fiebert IM, Spielholz NI, Applegate EB, Fox C, Jaro J, Joel L. Comparison of EMG activity of medial and lateral hamstrings during isometric contractions at various cuff weight loads. Knee. 2001;8(2):145-150. doi: 10.1016/S0968-0160(00)00079-X

8. Worrell TW, Karst G, Adamczyk D, Moore R, Stanley C, Steimel B, et al. Influence of joint position on electromyographic and torque generation during maximal voluntary isometric contractions of the hamstrings and gluteus maximus muscles. J Orthop Sports Phys Ther. 2001;31(12):730-740. doi: 10.2519/jospt.2001.31.12.730 pmid: 11767248

9. Mohamed O, Perry J, Hislop H. Relationship between wire EMG activity, muscle length, and torque of the hamstrings. Clin Biomech (Bristol, Avon). 2002;17(8):569-579. doi: 10.1016/S0268-0033(02)00070-0

10. Albertus-Kajee Y, Tucker R, Derman W, Lamberts RP, Lambert MI. Alternative methods of normalising EMG during running. J Electromyogr Kinesiol. 2011;21(4):579-586. doi: 10.1016/j.jelekin.2011.03.009 pmid: 21531148

11. Fauth ML, Petushek EJ, Feldmann CR, Hsu BE, Garceau LR, Lutsch BN, et al. Reliability of surface electromyography during maximal voluntary isometric contractions, jump landings, and cutting. J Strength Cond Res. 2010;24(4):1131-1137. doi: 10.1519/JSC.0b013e3181cc2353 pmid: 20179648

12. Onishi H, Yagi R, Oyama M, Akasaka K, Ihashi K, Handa Y. EMG-angle relationship of the hamstring muscles during maximum knee flexion. J Electromyogr Kinesiol. 2002;12(5):399-406. doi: 10.1016/S10506411(02)00033-0

13. Callaghan MJ, Oldham JA. A Critical Review of Electrical Stimulation of the Quadriceps Muscles. Critical Rev Physic Rehabilit Med. 1997;9(3-4):301-314. doi: 10.1615/CritRevPhysRehabilMed.v9.i3-4.60

14. Selkowitz DM. Improvement in isometric strength of the quadriceps femoris muscle after training with electrical stimulation. Phys Ther. 1985;65(2):186-196. doi: 10.1093/ptj/65.2.186 pmid: 3871529

15. Lim W. Easy method for measuring stretching intensities in real clinical settings and effects of different stretching intensities on flexibility. J Back Musculoskelet Rehabil. 2018. doi: 10.3233/BMR-181243 pmid: 30530964

16. Yin L, Chen K, Guo L, Cheng L, Wang F, Yang L. Identifying the Functional Flexion-extension Axis of the Knee: An In-Vivo Kinematics Study. PLoS One. 2015;10(6):e0128877. doi: 10.1371/journal.pone.0128877 pmid: 26039711

17. Perotto AO. Anatomical guide for the electromyographer: the limbs and trunk.: Charles $\mathrm{C}$ Thomas Publisher; 2011.

18. SENIAM [Internet]. Surface ElectroMyoGraphy for the Non-Invasive Assessment of Muscles. 2020. Available from: http://www.seniam.org/.

19. Fiebert IM, Roach KE, Fingerhut B, Levy J, Schumacher A. EMG activity of medial and lateral hamstrings at three positions of tibial rotation during low-force isometric knee flexion contractions. J Back Musculoskelet Rehabil. 1997;8(3):215-222. doi: 10.3233/BMR-1997-8306 pmid: 24572822

20. Jonasson G, Helgason A, Ingvarsson T, Kristjansson AM, Briem K. The Effect of Tibial Rotation on the Contribution of Medial and Lateral Hamstrings During Isometric Knee Flexion. Sports Health. 2016;8(2):161-166. doi: 10.1177/1941738115625039 pmid: 26721286

21. Beyer EB, Lunden JB, Russell Giveans M. Medial and Lateral Hamstrings Response and Force Production at Varying Degrees of Knee Flexion and Tibial Rotation in Healthy Individuals. Int J Sports Phys Ther. 2019;14(3):376-383. doi: 10.26603/ijspt20190376 pmid: 31681497

22. Johnson AJP. A two-method comparison of muscle testing the serratus anterior: Daniels and Worthingham vs. Kendall and McCreary [PhD Thesis]. University of North Dakota; 1993.

23. Perry J, Easterday CS, Antonelli DJ. Surface versus intramuscular electrodes for electromyography of superficial and deep muscles. Phys Ther. 1981;61(1):7-15. doi: 10.1093/ptj/61.1.7 pmid: 7454803

24. Ekstrom RA, Soderberg GL, Donatelli RA. Normalization procedures using maximum voluntary isometric contractions for the serratus anterior and trapezius muscles during surface EMG analysis. J Electromyogr Kinesiol. 2005;15(4):418-428. doi: 10.1016/j.jelekin.2004.09.006 pmid: 15811612

25. Smith J, Padgett DJ, Kaufman KR, Harrington SP, An K-N, Irby SE. Rhomboid muscle electromyography activity during 3 different manual muscle tests. Arch Phys Med Rehabil. 2004;85(6):987-992. doi: 10.1016/S00039993(03)00618-X

26. Boettcher CE, Ginn KA, Cathers I. Standard maximum isometric voluntary contraction tests for normalizing shoulder muscle EMG. J Orthop Res. 2008;26(12):1591-1597. doi: 10.1002/jor.20675 pmid: 18528827

27. Mohamed O, Perry J, Hislop H. Synergy of medial and lateral hamstrings at three positions of tibial rotation during maximum isometric knee flexion. Knee. 2003;10(3):277-281. doi: 10.1016/S0968-0160(02)00140-0 
28. Enoka RM. Muscle strength and its development. New perspectives. Sports Med. 1988;6(3):146-168. doi: 10.2165/00007256-198806030-00003 pmid: 3055145

29. Hughes RE, Chaffin DB, Lavender SA, Andersson GB. Evaluation of muscle force prediction models of the lumbar trunk using surface electromyography. J Orthop Res. 1994;12(5):689-698. doi: 10.1002/jor.1100120512 pmid: 7931786

30. Adouni M, Shirazi-Adl A, Marouane H. Role of gastrocnemius activation in knee joint biomechanics: gastrocnemius acts as an ACL antagonist. Comput Methods Biomech Biomed Engin. 2016;19(4):376-385. doi: 10.1080/10255842.2015.1032943 pmid: 25892616

31. Worrell TW, Denegar CR, Armstrong SL, Perrin DH. Effect of body position on hamstring muscle group average torque. J Orthop Sports Phys Ther. 1990;11(10):449-452. doi: 10.2519/jospt.1990.11.10.449 pmid: 18796892 